\title{
New Hyperkähler Metrics and New Supermultiplets *
}

\author{
U. Lindström ${ }^{\star \star}$ and M. Roček
}

Institute for Theoretical Physics, State University of New York at Stony Brook, Stony Brook, NY 11794-3840, USA

\begin{abstract}
We present new constructions of hyperkähler metrics along with the three new classes of $N=4$ supermultiplets that they derive from. Further, we provide a general setting for understanding the constructions and give a detailed description of the multiplets in $N=2$ and $N=4$ superspace.
\end{abstract}

\section{Introduction}

In this paper we present a general class of new Legendre transform constructions of hyperkähler metrics in a broad framework which illuminates our previous constructions [1-3]. As in these references, we construct (two or three dimensional) $N=4$ supersymmetric nonlinear $\sigma$-models in terms of off-shell multiplets and then find a duality relation via a Legendre transform to a formulation in terms of $N=2$ chiral superfields $[4,5]$. This yields the Kähler potential, and hence the metric, explicitly. In contrast with the original Legendre transform construction, the methods presented here do not in general lead only to metrics with isometries or analogous constraints. Our basic new insight is an understanding of $N=4$ superspace that allows us to find broad new classes of off-shell multiplets. We use the holomorphic superspace techniques of $[4,6,7,3]$; the method is presumably equivalent to the harmonic superspace approach $[8,9]$ (which, however, lacks a general technique for extracting the metric), and is closely related to twistor theory $[10,2]$.

In Sect. II, we give three general classes of constructions without explicit reference to superspace or supersymmetry. In Sect. III, we discuss $N=4$ superspace and the three classes of supermultiplets that lead to the constructions of Sect. II; in preparation for finding the metric, we describe these multiplets in $N=2$ superspace. Finally, in Sect. IV we derive the construction of Sect. II. We use the notation of $[2,3]$ where applicable.

\footnotetext{
* Supported in part by NSF grant No. PHY 85-07627
}

$\star \star$ On leave of absence from ITP, University of Stockholm 


\section{Construction of New Hyperkähler Metrics}

In this section, we present our new constructions. We begin with a description of a general setting that applies to all of them and then give them explicitly. We focus on four (real) dimensional manifolds, but the generalization to higher dimensions is obvious and involves no complications.

We start with a meromorphic map $\eta(\zeta)$ of the Riemann sphere into itself, and define a real structure $R: \eta(\zeta) \rightarrow \overline{\eta(-1 / \bar{\zeta})}$ (complex conjugation composed with the antipodal map). We fix the dependence of $\eta$ on $\zeta$ and work in the resulting parameter space with coordinates $\left\{w_{a}\right\}$; different choices of dependence lead to different constructions. We then consider a function $F\left(w_{a}\right)$ :

$$
F\left(w_{a}\right)=\frac{1}{2 \pi i} \oint_{C} \frac{d \zeta}{\zeta} G(\eta, \zeta)
$$

where $G$ is an arbitrary analytic function of its arguments, and $C$ is an appropriately chosen contour. The parameter space is reduced to a four (real) dimensional space by extremizing $F\left(w_{a}\right)$ with respect to all but four of its arguments. The Kähler potential is finally constructed as a (possibly nonlinear) Legendre transform of $F$ considered as a function on the reduced space. We now turn to the three classes of constructions that we have obtained. For the higher dimensional case, these constructions can be combined freely.

\section{(a) Generalized Linear $\eta(\zeta)$}

In this subsection we generalize the linear Legendre transform construction $[1,5,2]$. We consider $\zeta^{n} \eta(\zeta)$ to be a polynomial of order $2 n$ in $\zeta$ satisfying the reality condition $R \eta=\eta$ :

$$
\eta(\zeta) \equiv \sum_{a=-n}^{n} w_{a} \zeta^{a}, \quad w_{-a}=(-)^{a} \bar{w}_{a} .
$$

Note that $w_{0}$ is real. We define $F\left(w_{a}\right)$ as in (2.1); then $F$ satisfies

$$
F_{a b}=F_{(a+c)(b-c)}, \text { where } F_{a} \equiv \frac{\partial F}{\partial w_{a}} \text {, etc. }
$$

Next we let $z \equiv w_{n}, v \equiv w_{n-1}$. We extremize with respect to the remaining $\left\{w_{\underline{a}}, 2-n \leqq \underline{a} \leqq n-2\right\}$, and do a Legendre transform with respect to $v$ and $\bar{v}$; then the Kähler potential $K$ is

$$
K(z, \bar{z}, u, \bar{u})=F\left(z, \bar{z}, v, \bar{v}, w_{\underline{a}}, \bar{w}_{\underline{a}}\right)-u v-\bar{u} \bar{v},
$$

where

$$
v=v(z, \bar{z}, u, \bar{u}), \quad w_{\underline{a}}=w_{\underline{a}}(z, \bar{z}, u, \bar{u}),
$$

are solutions to

$$
F_{v}=u, \quad F_{\underline{a}}=0
$$

For $n=1$, this is precisely the linear Legendre transform construction. The metric is constructed as usual from the Kähler potential, and can be expressed in terms 
of derivatives of the function $F$ as follows: Differentiating (2.6), we find the linear systems of equations

$$
\begin{aligned}
& \mathbb{M} \frac{\partial}{\partial z} \mathbb{V}=-\frac{\partial}{\partial z} \nabla F, \\
& \mathbb{M} \frac{\partial}{\partial \bar{z}} \mathbb{V}=-\frac{\partial}{\partial \bar{z}} \nabla F, \\
& \mathbb{M} \frac{\partial}{\partial u} \mathbb{V}=\left(\begin{array}{c}
1 \\
0 \\
\vdots \\
0
\end{array}\right), \\
& \mathbb{M} \frac{\partial}{\partial \bar{u}} \mathbb{V}=\left(\begin{array}{c}
0 \\
1 \\
0 \\
\vdots \\
0
\end{array}\right),
\end{aligned}
$$

where

$$
\begin{gathered}
\mathbb{M} \equiv\left(\begin{array}{ccc}
F_{v v} & F_{v \bar{v}} & F_{v \underline{a}} \\
F_{v \bar{v}} & F_{\bar{v} \bar{v}} & F_{\bar{v} \underline{a}} \\
F_{\underline{b} v} & F_{\underline{b} \bar{v}} & F_{\underline{b} \underline{a}}
\end{array}\right), \\
\mathbb{V} \equiv\left(\begin{array}{c}
v \\
\bar{v} \\
w_{2-n} \\
\vdots \\
w_{n-2}
\end{array}\right), \quad \nabla F \equiv\left(\begin{array}{c}
F_{v} \\
F_{\bar{v}} \\
F_{2-n} \\
\vdots \\
F_{n-2}
\end{array}\right) .
\end{gathered}
$$

These equations express the metric in terms of second derivatives of $F$ :

$$
\begin{gathered}
K_{u \bar{u}}=-v_{\bar{u}}=-(10 \ldots 0) \mathbb{M}^{-1}\left(\begin{array}{c}
0 \\
1 \\
0 \\
\vdots \\
0
\end{array}\right), \\
K_{u \bar{z}}=-v_{\bar{u}}=(10 \ldots 0) \mathbb{M}^{-1} \frac{\partial}{\partial \bar{z}} \nabla F, \\
K_{z \bar{z}}=F_{z \bar{z}}-\left(\frac{\partial}{\partial z} \dot{\nabla} F\right) \mathbb{M}^{-1} \frac{\partial}{\partial \bar{z}} \nabla F .
\end{gathered}
$$

In the nonholomorphic coordinates $z, \bar{z}, v, \bar{v}$ found by reversing the Legendre transform, modulo the restriction to the hypersurface $F_{a}=0$, the metric is thus found completely explicitly. As far as we can see, only the $n=1$ case has an isometry. 
(b) Generalized Nonlinear $\eta(\zeta)$

In this subsection we generalize the nonlinear Legendre transform construction [3]. We consider $\eta(\zeta)=P(\zeta) / Q(\zeta)$, where $P$ and $Q$ are polynomials of order $n$ in $\zeta$. We require $\eta$ to satisfy the reality condition $R \eta=(-)^{n} \eta^{-1}$ :

$$
\eta(\zeta)=\frac{\bar{z}+\sum_{a=1}^{n-1} \zeta^{a} w_{a}+\zeta^{n} e^{i \theta}}{1+\sum_{a=1}^{n-1}(-\zeta)^{a} \bar{w}_{n-a} e^{i \theta}+(-\zeta)^{n} z e^{i \theta}} .
$$

Note that without loss of generality we have chosen the denominator to begin with 1 , and that $\theta$ is real. For $n$ even, we could have used the simpler reality condition of subsection $(a): R \eta^{\prime}=\eta^{\prime}, \eta^{\prime} \equiv i(1+\eta) /(1-\eta)$. However, this is not possible for $n$ odd, and for the sake of uniformity of notation, we work with (2.10). Again we define $F\left(w_{a}\right)$ as in (2.1); then $F$ satisfies certain differential conditions. We define $\bar{v} \equiv w_{1}+\bar{w}_{n-1} e^{i \theta}$, and, as above, extremize with respect to $\left\{w_{a}, 2 \leqq a \leqq n-2\right\}$ and do a Legendre transform with respect to $v$ and $\bar{v}$. Then the Kähler potential $K$ is precisely (2.4) with the conditions (2.5) and (2.6) above. The Legendre transform is linear except for $n=1$ (the case analyzed in detail in [3]), as then $v$ and $\bar{v}$ are not independent. As in the linear case above $(2.7,8.9)$, the metric can be expressed in terms of second derivatives of $F$, and found explicitly in nonholomorphic coordinates. As far as we can see, the metrics constructed in this way have no isometries for any $n$, though the $n=1$ case is special [3].

(c) General Holomorphic $\eta(\zeta)$

In this subsection we consider an arbitrary holomorphic $\eta(\zeta)$ :

$$
\eta(\zeta)=\sum_{a=0}^{\infty} w_{a} \zeta^{a},
$$

and we impose no particular condition on $R \eta$. We define $F\left(w_{a}\right)$ as in (2.1) except that $G(\eta, \zeta)$ is replaced by $G(\eta, R \eta, \zeta)$ (since in the previous examples $R \eta$ was expressed in terms of $\eta$, this is a natural generalization). As in the general linear case, we identify $\bar{z}=w_{0}, \bar{v}=w_{1}$, and find the Kähler potential by performing a Legendre transform with respect to $v$ and $\bar{v}$ and extremizing with respect to $w_{a}, \underline{a} \geqq 2$. Since this gives an infinite system of equations, the construction is less well defined than the ones above. However, because $\eta$ and $R \eta$ are algebraically independent, the holomorphic construction is in some sense the most general. In particular, at least formally, any of the generalized linear constructions of subsection $(a)$ can be rewritten in the holomorphic language (see below).

\section{New Supermultiplets}

In this section, we describe the new supermultiplets in $N=4$ and 2 superspace. In the next section, we use these multiplets to derive the constructions of Sect. II. We begin with a description of our approach to $N=4$ superspace; this provides a general setting that applies to all the new multiplets. 
The $N=4$ superspace is (two or three dimensional) Minkowski space enlarged by a complex isodoublet anticommuting spinor coordinate $\left(\theta^{a \alpha}, \bar{\theta}_{a}^{\alpha}\right)$. We use corresponding spinor derivatives that obey

$$
\left\{D_{a \alpha}, \bar{D}_{\beta}^{b}\right\}=i \delta_{a}^{b} \partial_{\alpha \beta}, \quad\left\{D_{a \alpha}, D_{b \beta}\right\}=0,
$$

where $\partial_{\alpha \beta}$ is the usual spacetime derivative in spinor notation. We often reduce to $N=2$ superspace and identify $\mathscr{D} \equiv D_{1}$ as the $N=2$ spinor derivative and $D_{2}$ as the generator of the nonmanifest supersymmetries.

In $N=4$ superspace we work with scalar superfields that are totally symmetric representations of $S U(2)$,

$$
\Phi_{a_{1} \ldots a_{n}}=\frac{1}{n !} \Phi_{\left(a_{1} \ldots a_{n}\right)} .
$$

A manifestly $N=4$ supersymmetric measure in superspace is $d^{D} x d^{4} \theta d^{4} \bar{\theta}=$ $d^{D} x D^{4} \bar{D}^{4}$, which has dimension $4-D$, and hence is unsuitable for $\sigma$-model actions. As described in $[4,5,7,3]$, we introduce a subspace of superspace (analogous to the chiral subspaces of $N=2$ superspace) parametrized by a complex coordinate $\zeta$ on the Riemann sphere. We believe that this is essentially equivalent to the Harmonic superspace approach of $[8,9]$, although we have not worked out the precise relation. We define a projective isospinor $u^{a}=(1, \zeta)$ and define the subspace using

$$
\nabla_{\alpha}=u^{a} D_{a \alpha}, \quad \bar{\nabla}_{\alpha}=u^{a} \bar{D}_{\alpha}^{b} \varepsilon_{a b} \equiv u_{a} \bar{D}_{\alpha}^{a} .
$$

The measure on the subspace is defined by the orthogonal operators

$$
\begin{aligned}
\Delta_{\alpha} & =v^{a} D_{a \alpha}, & \bar{\Delta}_{\alpha} & =v_{a} \bar{D}_{\alpha}^{a}, \\
v_{a} u^{a} & =2, & v^{a} & =\left(\zeta^{-1},-1\right) \Leftrightarrow v_{a}=\left(1, \zeta^{-1}\right)
\end{aligned}
$$

and is

$$
(32 \pi i)^{-1} \oint_{C} \zeta d \zeta d^{D} \times \Delta^{2}(\zeta) \bar{\Delta}^{2}(\zeta)
$$

The algebra (3.1) becomes

$$
\begin{aligned}
\{\nabla, \nabla\} & =\{\Delta, \Delta\}=\{\nabla, \bar{\nabla}\}=\{\Delta, \bar{\Delta}\}=\{\nabla, \Delta\}=0, \\
\left\{\nabla_{\alpha}, \bar{\Delta}_{\beta}\right\} & =-\left\{\bar{\nabla}_{\alpha}, \Delta_{\beta}\right\}=2 i \partial_{\alpha \beta} .
\end{aligned}
$$

The real structure $R$ acts on the subspace: $R \nabla=-(\zeta)^{-1} \bar{\nabla}, R \Delta=-\zeta \bar{\Delta}$. We further saturate the indices on the superfields (3.2) with $u^{a}$ s to obtain the superfields

$$
\Phi(\zeta)=u^{a_{1}} \ldots u^{a_{n}} \Phi_{a_{1} \ldots a_{n}} .
$$

The basic superfields $\eta(\zeta)$ that live in the subspace are constructed out of superfields such as (3.7) and are constrained to satisfy

$$
\nabla \eta(\zeta)=\bar{\nabla} \eta(\zeta)=0
$$

A Lagrange density constructed out of such superfields also obeys the constraint (3.8), and thus can be integrated with the measure (3.5) to get a fully $N=4$ supersymmetric action. For such actions, the measure can be reduced to $N=2$ 
superspace as follows [6]: We write

$$
\Delta=\zeta^{-1}(2 \mathscr{D}-\nabla), \quad \bar{\Delta}=2 \overline{\mathscr{D}}+\zeta^{-1} \bar{\nabla} .
$$

Because the Lagrange density obeys the constraints (3.8), the measure (3.5) becomes

$$
\frac{1}{2 \pi i} \oint_{C} \frac{d \zeta}{\zeta} d^{D} x \mathscr{D}^{2} \overline{\mathscr{D}}^{2} \text {. }
$$

The superfields $\eta(\zeta)$ are expressed in terms of $N=2$ superfields by expanding the constraints (3.8) in powers of $\zeta$ and separating those that give $N=2$ constraints from those that express $D_{2}$ in terms of $\mathscr{D}$, and hence give the nonmanifest supersymmetries.

This summarizes the general context for our new multiplets. We now present three cases that differ by their $\zeta$-dependence and by the reality conditions they satisfy.

\section{(a) Generalized Linear Multiplets}

We start with a real $N=4$ supermultiplet (3.2); since $S U(2)$ representations are real only for integer isospin, we have an even number $2 n$ of indices on $\Phi$ :

$$
\Phi_{a_{1} \ldots a_{2 n}}=\varepsilon_{a_{1} b_{1}} \ldots \varepsilon_{a_{2 n} b_{2 n}} \bar{\Phi}^{b_{1} \ldots b_{2 n}}
$$

The basic superfield satisfying the constraints (3.8) is $\eta(\zeta)$ :

$$
\eta(\zeta)=\zeta^{-n} u^{a_{1}} \ldots u^{a_{2 n}} \Phi_{a_{1} \ldots a_{2 n}}=\sum_{m=-n}^{n}\left[\begin{array}{l}
2 n \\
m+n
\end{array}\right] \zeta^{m} \Phi_{m+n},
$$

where $\Phi_{p}$ is the component with exactly $p$ of the $a$ 's $=2$. Because $\Phi$ is real $\left(\Phi_{p}=(-)^{p} \overline{\Phi_{2 n-p}}\right)$, the real structure $R$ sends $\eta$ into itself (cf. Subsect. IIa). The constraints (3.8) to the subspace imply that $\Phi$ satisfies:

$$
D_{(b} \Phi_{\left.a_{1} \ldots a_{2 n}\right)}=0, \quad \bar{D}_{(b} \Phi_{\left.a_{1} \ldots a_{2 n}\right)}=0
$$

(for $n=1$ these are the familiar constraints of the $N=4$ linear multiplet). In terms of $\Phi_{p}$, these are

$$
(p+1) D_{2} \Phi_{p}+(2 n-p) D_{1} \Phi_{p+1}=0,
$$

as well as the complex conjugate. As sketched above, an $N=4$ supersymmetric action is given by:

$$
I=\int d^{D} x \oint_{C} \frac{\zeta d \zeta}{32 \pi i} \Delta^{2} \bar{\Delta}^{2} G(\eta, \zeta)
$$

for $G(\eta, \zeta)$ an arbitrary analytic function.

The $N=2$ reduction of the constraints (3.14) is

$$
\begin{aligned}
\mathscr{D} \Phi_{0} & =0 \\
D_{2} \Phi_{0} & =2 n \mathscr{D} \Phi_{1} \Rightarrow \mathscr{D}^{2} \Phi_{1} \equiv \mathscr{D}^{2} \bar{\Sigma}=0, \\
D_{2} \Phi_{p} & =\left(\frac{2 n-p}{p+1}\right) \mathscr{D} \Phi_{p+1}, \quad p>0 .
\end{aligned}
$$


Thus $\Phi_{0}$ is an antichiral $N=2$ superfield, $\Phi_{1}$ is a complex linear $N=2$ superfield, and the remaining superfields are unconstrained. The constraints involving $D_{2}$ are the nonmanifest $N=4$ supersymmetry transformations in $N=2$ language. For $n=1, \Phi_{1}$ is a real linear $N=2$ superfield ( $\Phi_{n}$ is always real). The $\Phi_{p}$ for $1<p<2 n-1$ are auxiliary superfields with algebraic equations of motion. Finally, we emphasize that these multiplets are not the relaxed hypermultiplet of [12] or their generalizations in harmonic superspace [9], as we can write meaningful actions without Lagrange multiplier multiplets.

\section{(b) Generalized Nonlinear Multiplets}

We start with a complex $N=4$ supermultiplet (3.2). The basic superfield satisfying the constraints $(3.8)$ is $\eta(\zeta)$ :

$$
\eta(\zeta)=\frac{u^{a_{1}} \ldots u^{a_{n}} \Phi_{a_{1} \ldots a_{n}}}{u_{a_{1}} \ldots u_{a_{n}} \bar{\Phi}^{a_{1} \ldots a_{n}}}
$$

This $\eta$ satisfies the reality condition $R \eta=(-)^{n} \zeta^{-1}$. Because $\eta$ is independent of the scale of $\Phi$, we can fix this; by analogy to the $n=1$ case, we choose

$$
\Phi_{a_{1} \ldots a_{n}} \bar{\Phi}^{a_{1} \ldots a_{n}}=1
$$

The constraints (3.8) imply that $\Phi$ satisfies

$$
\Phi_{\left(a_{1} \ldots a_{n}\right.} D_{a_{n+1}} \bar{\Phi}_{\left.a_{n+2} \ldots a_{2 n+1}\right)}=\bar{\Phi}_{\left(a_{1} \ldots a_{n}\right.} D_{a_{n+1}} \Phi_{\left.a_{n+2} \ldots a_{2 n+1}\right)},
$$

where the indices on $\bar{\Phi}$ have been lowered with the $S U(2)$ symplectic metric $\varepsilon$. (For $n=1$, these are the familiar constraints of the nonlinear multiplet [11].) The action is precisely the same as for the generalized linear case (3.15).

Without loss of generality, we can divide $\Phi$ and $\bar{\Phi}$ by $\Phi$ and $\bar{\Phi}^{n}$; then the $N=2$ reduction gives

$$
\mathscr{D}\left(\frac{\Phi_{0}}{\bar{\Phi}^{n}}\right)=0, \quad \mathscr{D}^{2}\left(\frac{\bar{\Phi}^{1} \Phi_{n}+\Phi_{1} \bar{\Phi}^{n}}{\left(\bar{\Phi}^{n}\right)^{2}}\right) \equiv \mathscr{D}^{2} \bar{\Sigma}=0
$$

as well as constraints that give the nonmanifest supersymmetry transformations. Again, as for the generalized linear case, for $n>1$, we find an antichiral and a complex linear superfield, and further auxiliary superfields. (The $n=1$ case is special [3].)

\section{(c) Holomorphic Multiplet}

The holomorphic multiplet is a natural extension of the generalized linear multiplet. The basic superfield satisfying the constraints (3.8) is now given by a holomorphic function $\eta(\zeta)$. We impose no reality condition. The general $N=4$ supersymmetric action is now given as in (3.15), but with an arbitrary analytic function $G$ which depends on both $\eta$ and $R \eta$ (and $\zeta$ ). As in the generalized linear case, after reducing to $N=2$ superspace we find that $\Phi_{0}$ is antichiral, $\Phi_{1}$ is complex linear, and all of the other superfields are auxiliary. We believe that this multiplet is closely related to the complex analytic multiplet $q^{+}$of $[8,9]$. 


\section{Origin of the New Construction of Hyperkähler Metrics}

With the tools assembled in the previous section, it is simple to derive the constructions of Sect. II. We start with the $N=2$ superspace reduction of the action (3.15),

$$
\int d^{D} x \mathscr{D}^{2} \overline{\mathscr{D}}^{2} \oint_{C} \frac{d \zeta}{2 \pi i \zeta} G(\eta, \zeta)
$$

and relax the constraint on the complex linear superfield $\Sigma$ by introducing a chiral Lagrange multiplier (cf. $[1,2,3]$ ). This leads to a two-dimensional Legendre transform with respect to $\Sigma$ and $\bar{\Sigma}$. The Kähler potential is found by performing the Legendre transform and eliminating the auxiliary superfields. This elimination can be done, without changing the result, before, after, or simultaneously with the Legendre transform. The constructions of Sect. II follow after the appropriate identification of coordinates and superfields:

$$
\begin{aligned}
& \text { (a) }\left[\begin{array}{l}
2 n \\
a+n
\end{array}\right] \Phi_{a+n} \leftrightarrow w_{a}, \\
& \text { (b) } \frac{\Phi_{0}}{\bar{\Phi}^{n}} \bar{z}, \quad\left[\begin{array}{l}
n \\
a
\end{array}\right] \Phi_{a} \leftrightarrow w_{a}, \quad \frac{\Phi_{n}}{\bar{\Phi}^{n}} \leftrightarrow e^{i \theta}, \\
& \text { (c) } a ! \Phi_{a} \leftrightarrow w_{a} .
\end{aligned}
$$

The duality between chiral and complex linear superfields that plays a crucial role in these constructions can be used to give a relation between the generalized linear constructions and particular cases of the holomorphic construction. We consider an action in terms of a particular generalized linear multiplet $\eta_{2 n} \equiv \sum_{-n}^{n} \zeta^{a} \eta_{a}$, replace it by a multiplet $\ell \equiv \sum_{-\infty}^{\infty} \zeta^{a} \ell_{a}=R \ell$ with arbitrary $\zeta$ dependence, and introduce a holomorphic Lagrange multiplier field $\eta_{H} \equiv \sum_{0}^{\infty} \zeta^{a} \eta_{a}$ :

$$
\int d^{D} x \mathscr{D}^{2} \bar{D}^{2} \oint_{C} \frac{d \zeta}{2 \pi i \zeta}\left[G(\ell, \zeta)+\left(\zeta^{n} \eta_{H}+(-\zeta)^{-n} R \eta_{H}\right) \ell\right] .
$$

Varying the auxiliary components of $\eta_{H},\left\{\eta_{a}, a>1\right\}$, sets all $\left\{\ell_{a},|a|>n+1\right\}$ to zero, whereas varying the antichiral and linear components $\eta_{0}$ and $\eta_{1}$ constrains $\ell_{1-n}$ and $\ell_{-n}$ to be linear and antichiral respectively; thus varying $\eta_{H}$ restricts $\ell$ to $\eta_{2 n}$. On the other hand, one may vary $\ell$ instead and arrive at an action in terms of $\eta_{H}$. Though formally one may rewrite all the generalized linear actions in holomorphic terms through this procedure, it is difficult to see how to make sense of it for Lagrangians such as $\eta \ln \eta[6,7,2]$ when the contour encloses singularities other than $\zeta=0$.

This concludes our derivation of the constructions of Sect. II. The generalization to higher dimensional hyperkähler metrics is obvious, and can use an arbitrary mix of the various multiplets discussed here. The constructions are local; the outstanding problem is to find a classification of the complete metrics that can be 
constructed using these methods, and in particular, to determine whether compact examples such as the K3 surface are among them.

Acknowledgements. We are happy to thank W. Nahm and B. Lawson for interesting discussions.

\section{References}

1. Lindström, U., Roček, M.: Scalar tensor duality and $N=1,2$ nonlinear $\sigma$-models. Nucl. Phys. B222, $285(1982)$

2. Hitchin, N. J., Karlhede, A., Lindström, U., Roček, M.: Hyperkähler metrics and supersymmetry. Commun. Math. Phys. 108, 535 (1987)

3. Karlhede, A., Lindström U., Roček, M.: Hyperkähler manifolds and nonlinear supermultiplets. Commun. Math. Phys. 108, 529 (1987)

4. Gates, S. J., Hull, C. M., Roček, M.: Twisted multiplets and new supersymmetric nonlinear $\sigma$-models. Nucl. Phys. B248, 157 (1984)

5. Howe, P. S., Karlhede, A., Lindström, U., Roček, M.: The geometry of duality. Phys. Lett. 168B, 89 (1986)

6. Karlhede, A., Lindström, U., Roček, M.: Self-interacting tensor multiplets in $N=2$ superspace. Phys. Lett. 147B, 297 (1984)

7. Grundberg, J., Lindström, U.: Actions for linear multiplets in six dimensions. Class. Quant. Grav. 2, L33 (1985)

8. Galperin, A., Ivanov, E., Kalitzin, S., Ogievietsky, V., Sokatchev, E.: Unconstrained $N=2$ matter Yang-Mills and supergravity theories in harmonic superspace. Class. Quant. Grav. 1, 496 (1984)

9. Galperin, A., Ivanov, E., Ogievietsky, V.: Duality transformations and most general matter self-coupling in $N=2$ supersymmetry. Nucl. Phys. B282, 74 (1987)

10. Penrose, R.: Nonlinear gravitons and curved twistor theory. Gen. Rel. Grav. 7, 31 (1976)

11. de Wit, B., van Holten, J. W., Van Proeyen, A.: Structure of $N=2$ supergravity. Nucl. Phys. B184, 77 (1981)

12. Howe, P. S., Stelle, K. S., Townsend, P. K.: The relaxed hypermultiplet: An unconstrained $N=2$ superfield theory. Nucl. Phys. B214, 519 (1983)

Communicated by L. Alvarez-Gaumé

Received June 21, 1987 
